\title{
FATORES DE RISCO DOS DIAGNÓSTICOS DE ENFERMAGEM DO DOMÍNIO SEGURANÇA/ PROTEÇÃO: REVISÃO INTEGRATIVA
}

\author{
Laísla Alves Moura ${ }^{1}$, Jéssica Naiara de Medeiros Araújo ${ }^{2}$, Danielly Oliveira Pitombeira ${ }^{3}$, Ana Paula Nunes de \\ Lima Fernandes ${ }^{4}$, Fabiane Rocha Botarelli ${ }^{5}$, Allyne Fortes Vitor ${ }^{6}$
}

RESUMO: Revisão integrativa com o objetivo de sintetizar as evidências disponíveis na literatura acerca dos fatores de risco dos diagnósticos de enfermagem do Domínio Segurança/Proteção e eventos adversos correlacionados em pacientes hospitalizados. Realizada em fevereiro de 2015 mediante busca nas bases de dados Cumulative Index to Nursing and Allied Health, Literatura Latino-americana e do Caribe em Ciências da Saúde, National Library of Medicine National Institutes of Health, Scientific Electronic Library Online e Scopus, que resultou em 19 estudos. Os fatores de risco mais prevalentes foram: tempo de internação prolongado, idade avançada e sobrecarga dos profissionais de enfermagem. A categoria Risco de lesão apresentou o maior número de fatores de risco sem correspondência NANDA Internacional (NANDA-I), em contraste, a categoria Risco de quedas apresentou o maior número de equivalentes. Deste modo, a presença de fatores de risco citados na literatura sem inclusão na NANDA-I denota necessidade de estudos mais aprofundados na temática.

DESCRITORES: Enfermagem; Segurança do paciente; Diagnóstico de enfermagem; Fatores de risco; Enfermagem baseada em evidências.

\section{RISK FACTORS OF THE NURSING DIAGNOSIS IN THE SAFETY/PROTECTION DOMAIN: INTEGRATIVE REVIEW}

ABSTRACT: Integrative review to summarize the available evidence in the literature on the risk factors for the nursing diagnoses in the Safety/ Protection Domain and correlated adverse events in hospitalized patients. Undertaken in February 2015 through a search in the databases Cumulative Index to Nursing and Allied Health, Literatura Latino-americana e do Caribe em Ciências da Saúde, National Library of Medicine National Institutes of Health, Scientific Electronic Library Online and Scopus, which resulted in 19 studies. The most prevalent risk factors were: extended hospitalization, advanced age and burden of nursing professionals. The category Risk for injury presented the largest number of risk factors without correspondence in NANDA International (NANDA-I), while the category Risk for falls presented the largest number of equivalents. Thus, the presence of the risk factors cited in the literature without inclusion in NANDA-I indicates the need for more in-depth studies on the theme. DESCRIPTORS: Nursing; Patient safety; Nursing diagnosis; Risk factors; Evidence-based nursing.

\section{FACTORES DE RIESGO DE LOS DIAGNÓSTICOS DE ENFERMERÍA DEL DOMINIO SEGURIDAD/PROTECCIÓN: REVISIÓN INTEGRADORA}

RESUMEN: Revisión integradora con objeto de sintetizar las evidencias disponibles en la literatura acerca de los factores de riesgo de los diagnósticos de enfermería del Dominio Seguridad/Protección y eventos adversos correlacionados en pacientes hospitalizados. Desarrollado en febrero del 2015 mediante búsqueda en las bases de datos Cumulative Index to Nursing and Allied Health, Literatura Latino-americana e do Caribe em Ciências da Saúde, National Library of Medicine National Institutes of Health, Scientific Electronic Library Online y Scopus, que resultó en 19 estudios. Los factores de riesgo más prevalentes fueron: tiempo de internación prolongado, edad avanzada y sobrecarga de los profesionales de enfermería. La categoría Riesgo de lesión presentó el mayor número de factores de riesgo sin correspondencia en NANDA Internacional (NANDA-I). En contraste, la categoría Riesgo de caídas presentó el mayor número de equivalentes. Así, la presencia de factores de riesgo citados en la literatura sin inclusión en la NANDA-I indica necesidad de estudios más a hondo en el tema.

DESCRIPTORES: Enfermería; Seguridad del paciente; Diagnóstico de enfermería; Factores de riesgo; Enfermería basada en evidencias.

${ }^{1}$ Enfermeira. Mestre em Enfermagem. Residente em Terapia Intensiva Adulto. Universidade Federal do Rio Grande do Norte. Natal, RN, Brasil.

${ }^{2}$ Enfermeira. Mestranda em Enfermagem. Universidade Federal do Rio Grande do Norte. Natal, RN, Brasil.

${ }^{3}$ Discente de Enfermagem. Universidade Federal do Rio Grande do Norte. Natal, RN, Brasil.

${ }^{4}$ Enfermeira. Doutoranda em Enfermagem. Universidade Federal do Rio Grande do Norte. Natal, RN, Brasil.

${ }^{5}$ Enfermeira. Doutoranda em Enfermagem. Docente de Enfermagem da Universidade Federal do Rio Grande do Norte. Natal, RN, Brasil.

${ }^{6}$ Enfermeira. Doutora em Enfermagem. Docente de Enfermagem da Universidade Federal do Rio Grande do Norte. Natal, RN, Brasil. 
Os Sistemas de Classificação referentes ao processo de enfermagem podem ser exemplificados pelas taxonomias NANDA Internacional (NANDA-I), Nursing Intervention Classification (NIC) e Nursing Outcomes Classification (NOC). A NANDA-I possui extensa abrangência clínica ao abordar os elementos essenciais à prática profissional, constituída pelos diagnósticos de enfermagem, dispostos em 13 domínios, 47 classes, e 217 diagnósticos de enfermagem (DE). Deste total, destaca-se o Domínio Segurança/Proteção cuja definição é estar livre de perigo, lesão física ou dano ao sistema imunológico; conservação contra perdas e proteção da segurança e da ausência de perigos, possui seis classes e $42 \mathrm{DE}^{(1)}$.

$\mathrm{Na}$ literatura, alguns destes DE podem ser correlacionados aos eventos adversos (EA), considerados como qualquer incidente evitável, associado ao cuidado ofertado durante a internação, caracterizado pela concessão de danos ao paciente(2).

Nesse âmbito, ao considerar a relevância dos EA no Brasil, o Ministério da Saúde (MS) vigorou a portaria 529/2013 que trata do Programa Nacional de Segurança do Paciente (PNSP) ${ }^{(2)}$, com o qual busca a implantação da gestão do risco e de núcleos de segurança do paciente em todos os estabelecimentos de saúde, priorizando as seguintes áreas: infecções, procedimentos cirúrgicos e de anestesiologia; prescrição, transcrição, dispensação e administração de medicamentos, sangue e hemoderivados; identificação de pacientes; comunicação no ambiente dos serviços; prevenção de quedas e úlceras por pressão; transferência de pacientes entre pontos de cuidado(2).

Ao tecer uma relação entre o teor da PNSP e a taxonomia da NANDA-I ${ }^{(1)}$, no concernente ao Domínio Segurança/ Proteção, destacam-se DE Risco de infecção, Risco de quedas, Risco de integridade da pele prejudicada, Risco de trauma e Risco de trauma vascular, ao serem considerados com elevada prevalência em estudo anterior ${ }^{(3)}$.

Na prática clínica, o enfermeiro pode inferir tais DE com o intuito de verificar os riscos e complicações aos quais os pacientes em regime de internação estão comumente expostos, além de gerenciar estes EA e elaborar intervenções individuais orientadas para sua prevenção.

As intervenções baseadas nas evidências científicas são inerentes ao Processo de Enfermagem à medida que auxiliam o enfermeiro no processo de tomada de decisão ${ }^{(1,4)}$. A Enfermagem Baseada em Evidência, por sua vez, é caracterizada pela avaliação minuciosa e rigorosa de estudos relevantes sobre um problema de enfermagem préestabelecido, a síntese dos dados expressivos e a obtenção de informações sobre novas intervenções, tratamentos e meios de prevenção, considera também a expertise do profissional e a vontade do paciente ${ }^{(5)}$.

Entretanto, existe a carência de estudos de análise de contexto e validação diagnóstica, o que limita os achados de evidências fortes que sustentem a prática diagnóstica na enfermagem ${ }^{(4)}$.

Destarte, justifica-se este estudo pelas recomendações estabelecidas pelo MS por meio da PNSP ao revelar a necessidade de estudos e pesquisas relacionadas à segurança do paciente, a fim de fortalecer as evidências científicas para direcionar a melhor prática assistencial e favorecer a efetividade da gestão de risco e eventos adversos.

Portanto, este estudo objetiva sintetizar as evidências disponíveis na literatura acerca dos fatores de risco dos diagnósticos de enfermagem do Domínio Segurança/Proteção e eventos adversos correlacionados em pacientes hospitalizados.

\section{- MÉTODO}

Trata-se de uma revisão integrativa (RI), método que possibilita incluir literatura teórica e empírica bem como estudos primários com diferentes abordagens metodológicas. Além do mais, permite gerar uma fonte de conhecimento atual sobre o problema e determinar se o conhecimento é válido para ser transferido para a prática ${ }^{(6)}$.

Com o intuito de operacionalizar esta investigação, foram executadas seis etapas: identificação do tema e seleção da questão norteadora; estabelecimento de critérios para inclusão e exclusão de estudo; definição das informações a serem extraídas/categorização dos estudos selecionados; avaliação dos estudos incluídos na 
revisão integrativa; interpretação dos resultados e apresentação da RI.

Para operacionalização desta revisão estabeleceu-se a seguinte questão norteadora: Quais as evidências disponíveis na literatura acerca dos fatores de risco dos diagnósticos de enfermagem do Domínio Segurança/ Proteção e eventos adversos correlacionados em pacientes hospitalizados?

A busca foi realizada em fevereiro de 2015 nas bases de dados Cumulative Index to Nursing and Allied Health (CINAHL), Literatura Latino-americana e do Caribe em Ciências da Saúde (LILACS), National Library of Medicine National Institutes of Health (PubMed), Scientific Electronic Library Online (Scielo) e Scopus mediante uso do proxy licenciado pela Universidade Federal do Rio Grande do Norte acessado via portal do Periódicos Capes. Para a captura dos artigos na LILACS e Scielo, foram executados cruzamentos de descritores e suas respectivas sinonímias indexados no DeCS (Descritores em Ciências da Saúde): segurança do paciente; enfermagem; fatores de risco. Além disso, usou-se a seguinte palavra-chave: evento adverso. Para busca na CINAHL, PubMed e Scopus utilizou-se os descritores e sinonímias correspondentes do MeSH (Medical Subjects Headings): Safety patient; nursing; risk factors; adverse effects. Os cruzamentos foram realizados como a aplicação do operador booleano AND.

Foram estabelecidos os seguintes critérios de inclusão: ser artigo completo de pesquisa primária, estar disponível nas bases de dados selecionadas e abordar os fatores de risco dos diagnósticos de enfermagem do Domínio Segurança/Proteção elencados ou eventos adversos correlacionados em pacientes ou profissionais de saúde em ambiente hospitalar. Para esta seleção, não foram instituídas restrição de idioma nem de data de publicação. Foram excluídos artigos de revisões, estudos de caso, carta ao editor, editoriais, teses e dissertações.

Portanto, foi procedido a aplicação do Teste de Relevância(7) em três rodadas por dois pesquisadores que abrangeram a aplicação de um protocolo contendo os critérios de inclusão/exclusão e a questão norteadora. Em caso de discordância, foi solucionada mediante consenso. Na primeira rodada, o teste foi empregado na leitura do título das publicações, e derivou 110 artigos; da segunda rodada, obteve-se 62 resumos e, por fim, como produto da terceira rodada, foram selecionados 19 artigos.

Para definir as informações a serem extraídas/categorização dos estudos, foi desenvolvido um formulário composto pelas seguintes informações: identificação do artigo original, características metodológicas do estudo, fatores de risco (FR) dos EA e a correlação destes com os FR dos DE da NANDA-I selecionados para esta investigação. Antes da leitura para extração das informações relevantes, executou-se uma listagem das possíveis sinonímias de expressões equivalentes ao FR dos DE do Domínio Segurança/Proteção da NANDA-I.

Em seguida, as informações extraídas foram categorizadas de acordo com a abordagem de cada DE previamente selecionado durante a leitura do texto. Assim, emergiram quatro categorias: Fatores de Risco para Risco de infecção; Fatores de Risco para quedas, Fatores de Risco para Risco de integridade da pele prejudicada; Fatores de Risco para Risco de lesão.

Para executar a avalição dos estudos, com referência à correspondência das evidências identificadas e os FR existentes na NANDA-I, utilizou-se a própria taxonomia como parâmetro para avaliação descritiva. Para descrever a força de evidência dos estudos, utilizou-se como referência a classificação do The Joanna Brigges Institute.

A interpretação dos resultados foi feita mediante a discussão com a literatura pertinente ao tema de estudo, e os resultados foram representados mediante uso de Tabelas.

\section{RESULTADOS}

Realizou-se uma análise descritiva e a síntese dos artigos selecionados ( $n=19)$ está apresentada na Tabela 1 e Tabela 2, a seguir.

Observa-se quanto à Tabela 1, que os estudos transversais foram os mais predominantes, do mesmo modo, a força de evidência VI foi a mais prevalente.

Tabela 1 - Distribuição das publicações, segundo a força de evidência e o delineamento do estudo. Natal, RN, Brasil, 2016 


\begin{tabular}{cccr} 
Força de evidência & Delineamento & Publicações & $\%$ \\
\hline \multirow{2}{*}{ IV } & Estudo de Coorte & 7 & 36,84 \\
\cline { 2 - 4 } & Caso-controle & 1 & 5,26 \\
\hline VI & Estudo Epidemiológico & 3 & 15,79 \\
\cline { 2 - 4 } & Transversal & 8 & 42,11 \\
\hline
\end{tabular}

Tabela 2 - Distribuição dos artigos conforme o país, ano de publicação, sujeitos e temática do estudo. Natal, RN, Brasil, 2016

\begin{tabular}{|c|c|c|}
\hline Variável & $\mathrm{N}$ & $\%$ \\
\hline \multicolumn{3}{|l|}{ País } \\
\hline Brasil & 12 & 63,16 \\
\hline Estados Unidos & 3 & 15,79 \\
\hline Outros & 4 & 21,05 \\
\hline Total & 19 & 100 \\
\hline \multicolumn{3}{|l|}{ Ano de publicação } \\
\hline Anteriores a 2009 & 1 & 5,26 \\
\hline 2009 & 3 & 15,79 \\
\hline 2011 & 5 & 26,31 \\
\hline 2012 & 8 & 42,11 \\
\hline 2013 & 2 & 10,53 \\
\hline Total & 19 & 100 \\
\hline \multicolumn{3}{|l|}{ Sujeitos } \\
\hline Idosos & 12 & 63,16 \\
\hline Adultos & 7 & 36,84 \\
\hline Total & 19 & 100 \\
\hline \multicolumn{3}{|l|}{ Temática do estudo } \\
\hline EA relacionados à administração de medicamento & 9 & 47,37 \\
\hline EA relacionados à Infecção & 1 & 5,26 \\
\hline EA relacionados a Quedas & 3 & 15,79 \\
\hline EA relacionados à categoria úlceras por pressão (UP) & 4 & 21,05 \\
\hline EA relacionados à UP e Quedas ou infecção & 2 & 10,53 \\
\hline Total & 19 & 100 \\
\hline
\end{tabular}

Na Tabela 2, identifica-se a maior prevalência de estudos no Brasil, realizados em sua maioria, no ano de 2012. Os estudos eram compostos em grande parte por idosos e os EA mais pesquisados estavam relacionados à administração de medicamentos.

Após o cruzamento das evidências com os fatores de risco correspondentes da NANDA-I, as mesmas foram categorizadas em Risco de infecção (Tabela 3), Risco de Quedas (Tabela 3), Risco de integridade da pele prejudicada (Tabela 4) e Risco de Lesão e Risco de trauma vascular (Tabela 5).

Na categoria Risco de infecção não foram encontrados correspondentes NANDA-I para os fatores de risco idade avançada, tempo de internação, sedação excessiva e nível de consciência diminuído. De modo semelhante, não houve correspondente NANDA-I para os FR sexo masculino, grades do leito abaixadas e sobrecarga de trabalho, referentes à categoria Risco de quedas.

Relativos à categoria Risco de integridade da pele prejudicada, não houve correspondente para os FR tempo de internação, internação em clínica cirúrgica, sobrecarga profissional e grau de dependência.

Quanto aos FR da categoria Risco de lesão/ Risco de trauma vascular, foram encontrados os respectivos correspondentes NANDA-I para omissão do procedimento, atrasos nos horários de administração, tempo de internação, inexistência de protocolos, ausência de educação continuada e falhas na anotação. 
Tabela 3 - Correspondência entre os Fatores de Risco para infecção e quedas identificados na literatura e os Fatores de Risco dos Diagnósticos de Enfermagem Risco de infecção e Risco de quedas. Natal, RN, Brasil, 2016

\begin{tabular}{|c|c|c|}
\hline \multicolumn{3}{|l|}{ Diagnóstico Risco de infecção } \\
\hline $\begin{array}{l}\text { Fatores de risco identificados na } \\
\text { literatura }\end{array}$ & Força da evidência & $\begin{array}{l}\text { Fator de Risco do Diagnóstico de Enfermagem Risco de } \\
\text { infecção correspondente }\end{array}$ \\
\hline Idade avançada & $\mathrm{VI}$ & Nenhum correspondente \\
\hline Tempo de internação > sete dias & $\mathrm{VI}$ & Nenhum correspondente \\
\hline Fumante ou ex-fumante & $\mathrm{VI}$ & Defesa primária inadequada \\
\hline Sedação excessiva & VI & Nenhum correspondente \\
\hline Nível de consciência diminuído & VI & Nenhum correspondente \\
\hline \multicolumn{3}{|l|}{ Diagnóstico Risco de Quedas } \\
\hline $\begin{array}{l}\text { Fatores de risco identificados na } \\
\text { literatura }\end{array}$ & Força da evidência & $\begin{array}{l}\text { Fator de risco do Diagnóstico de Enfermagem Risco de } \\
\text { quedas correspondente }\end{array}$ \\
\hline Sexo masculino & $\mathrm{VI}$ & Nenhum correspondente \\
\hline Doença neurológica & $\mathrm{VI}$ & Estado mental diminuído \\
\hline Grades do leito abaixadas & $\mathrm{VI}$ & Nenhum correspondente \\
\hline Idade avançada & $\mathrm{VI}$ & Idade acima de 65 anos \\
\hline Tempo de internação < 14 dias & IV & Quarto não familiar \\
\hline Histórico de quedas & IV & História de quedas \\
\hline Sobrecarga de trabalho & IV & Nenhum correspondente \\
\hline
\end{tabular}

Tabela 4 - Correspondência entre os FR para Úlcera por Pressão identificados na literatura e os FR do DE Risco de integridade da pele prejudicada. Natal, RN, Brasil, 2016

\begin{tabular}{lll} 
Fatores de risco identificados na literatura & $\begin{array}{l}\text { Força da } \\
\text { evidência }\end{array}$ & $\begin{array}{l}\text { Fatores de Risco do Diagnóstico de Enfermagem Risco de } \\
\text { integridade da pele prejudicada correspondente }\end{array}$ \\
\hline Tempo de internação $>$ 7 dias & VI & Nenhum correspondente \\
\hline Idade avançada & IV & Extremos de idade \\
\hline Tempo de cirurgia superior a 4h 30min & IV & Imobilização física \\
\hline Sobrecarga dos profissionais & IV & Nenhum correspondente \\
\hline Obesidade grave & IV & Estado nutricional desequilibrado \\
\hline Grau de dependência & VI & Nenhum correspondente
\end{tabular}

Tabela 5 - Correspondência entre os fatores de risco encontrados na literatura para erros de medicação e os FR dos DE Risco de lesão e Risco de trauma vascular. Natal, RN, Brasil, 2016 (continua)

\begin{tabular}{lll} 
Fatores de risco identificados na literatura & $\begin{array}{l}\text { Força da } \\
\text { evidência }\end{array}$ & $\begin{array}{l}\text { Fatores de risco dos diagnósticos de enfermagem risco de } \\
\text { lesão e risco de trauma vascular correspondentes }\end{array}$ \\
\hline Falha de verificação dos 9 certos & IV & Nenhum correspondente \\
\hline $\begin{array}{l}\text { Utilização inadequada de equipamentos } \\
\text { (bomba de infusão) }\end{array}$ & VI & Estrutura e organização do equipamento \\
\hline Falhas no preparo e/ou administração & IV & Concentração da solução \\
\hline Omissão do procedimento & IV & Nenhum correspondente \\
\hline Atrasos nos horários de administração & IV & Nenhum correspondente \\
\hline Tempo de internação > 7 dias & VI & Nenhum correspondente \\
\hline Ambiente inapropriado para preparo & VI & Estrutura e organização do local \\
\hline Conhecimento insuficiente & VI & Fatores cognitivos \\
\hline Impermeabilidade vascular & IV & Fixação inadequada do cateter \\
\hline Experiência profissional reduzida & VI & Fatores cognitivos \\
\hline Inexistência de protocolos & VI & Nenhum correspondente \\
\hline \hline
\end{tabular}




\begin{tabular}{lll}
\hline Sobrecarga dos profissionais & IV & Padrões de contratação de funcionário \\
\hline Ausência de programas de educação continuada & VI & Nenhum correspondente \\
\hline Falhas na anotação & VI & Nenhum correspondente
\end{tabular}

\section{- DISCUSSÃO}

Os EA são fatores que comprometem a assistência e apresentam potencial limitante ou incapacitante como consequência de um controle inadequado de riscos. Deste modo, é necessário enfatizar a necessidade de rastrear os fatores de risco envolvidos no desenvolvimento desses eventos e correlacioná-los aos DE do domínio Segurança/Proteção da NANDA-I com a finalidade de prevenir a sua ocorrência.

Assim, de acordo com os achados, o tempo de internação foi o fator de risco comum a todas as categorias. Concernente a este resultado, outra pesquisa ${ }^{(8)}$ estabeleceu a relação entre tempo de internação com a prevalência de $E A$, quando $77 \%$ da sua amostra desenvolveu infecção após um tempo médio de internação de cinco dias. $O$ tempo de internação prolongado expõe o paciente ao risco de sofrer com incidentes relacionados à assistência, como no caso dos erros de medicação e o surgimento de infecções, úlceras de pressão (UP) e outros ${ }^{(9-10)}$.

No contexto da prevalência de quedas no âmbito hospitalar, o tempo de internação prolongado surge como causa e consequência desse evento, pois os pacientes que têm internação prolongada estão mais propícios a quedas, do mesmo modo que o incidente aumentará o tempo de internação(11).

A idade avançada foi um fator de risco para EA prevalente entre os achados. Quanto a isso, sabe-se que as alterações fisiológicas do envelhecimento são capazes de tornar o organismo ainda mais vulnerável a doenças, lesões e complicações, especialmente durante a internação(12).

A sobrecarga profissional foi identificada como um facilitador da incidência de EA. De acordo com outro estudo $^{(13)}$, a elevada demanda de pacientes para um contingente insuficiente de enfermeiros aumenta expressivamente a ocorrências de incidentes associados à assistência. $\mathrm{O}$ presente estudo evidenciou que a excessiva carga de trabalho da equipe de enfermagem aumenta proporcionalmente a incidência de UP, quedas e erros de medicação, pois a razão entre as horas disponíveis de enfermagem para o quantitativo de profissionais relaciona-se diretamente à segurança do paciente e a assistência de qualidade ${ }^{(14)}$.

Quanto aos FR encontrados na literatura sem sinonímia na NANDA-(1), a maior desproporção foi encontrada na categoria Risco de lesão. O Risco de lesão é definido como resultado de condições ambientais interagindo com recursos adaptativos e defensivos do indivíduo(1) e esteve associado aos estudos sobre erro de medicação.

É valido ressaltar que todos os pacientes sob regime de internação estão sujeitos a risco(15). Destarte, a assistência de enfermagem sistematizada surge como meio de investigar esse risco de EA e a partir disso, intervir de modo a prevenir a sua incidência. A identificação adequada dos DE e seus respectivos fatores de risco constitui etapa fundamental para a eficácia das intervenções e na promoção da segurança do paciente.

$\mathrm{Na}$ categoria Risco de quedas, grades do leito abaixadas oferecem risco para a ocorrência do evento, no entanto não apresenta correspondente NANDA-I. As quedas tornaram-se um problema de saúde pública e um importante indicador da segurança do paciente, especialmente no tocante às atividades de enfermagem. Estimase que a cada ano, 424.000 pessoas morrem por quedas no mundo. É a segunda causa de morte não intencional, sucedida pelos acidentes de trânsito. Das quedas não fatais no contexto mundial, 37,3 milhões carecem de cuidados médicos ${ }^{(16)}$.

Assim, as quedas são EA e requerem atenção máxima em se tratando de pacientes hospitalizados. A medida preventiva de elevar as grades do leito é efetiva ao diminuir o risco e ainda é um indicador da assistência de enfermagem ${ }^{(17-18)}$.

Falhas no preparo, administração e ao conferir os nove certos estiveram relacionados a falhas assistenciais. Em um estudo desenvolvido em um hospital de grande porte no intuito de acompanhar o seguimento de todas as etapas referentes à administração de medicamentos, percebeu-se que a maior parte dos profissionais não confere medicação, hora ou paciente ${ }^{(19)}$. Deste modo, pode-se concluir que os EA relacionados à medicação estão relacionados à assistência de enfermagem e interferem diretamente nos indicadores de qualidade hospitalar e 
de segurança do paciente.

Ressalta-se, neste sentido, a importância da implementação da educação continuada e de protocolos capazes de padronizar o procedimento de administração de medicamentos. Os protocolos são ferramentas que contém as etapas específicas ordenadas de acordo com a sua execução e contribuem para a prevenção de equívocos ${ }^{(18)}$. Estas medidas podem contribuir expressivamente para reduzir a prevalência de eventos adversos e para a manutenção da segurança do paciente durante todo o período de internação.

\section{- CONCLUSÃO}

Como forma de sintetizar os resultados ora obtidos, considera-se que os fatores de risco relacionados aos $\mathrm{DE}$ Risco de infecção, Risco de quedas, Risco de integridade da pele prejudicada e Risco de lesão são clássicos no cenário hospitalar. Os Fatores de Risco mais prevalentes foram: tempo de internação prolongado, idade avançada e sobrecarga dos profissionais de enfermagem. Alguns FR não apresentaram correspondentes na NANDA-I para o DE correspondente.

A categoria Risco de lesão apresentou o maior número de FR sem correspondência NANDA-I, em contraste, a categoria Risco de quedas apresentou o maior número de equivalentes. Para tanto, o conhecimento proporcionado será importante para a prática de uma assistência voltada para a prevenção de eventos adversos relacionados aos DE em estudo, principalmente em pacientes internados em ambiente hospitalar.

Dessa forma, a presença de FR citados na literatura sem devida citação na NANDA-I denota a necessidade de estudos mais aprofundados na temática, como a análise de conceito e validação, ainda tão escassos na literatura.

\section{REFERÊNCIAS}

1. Herdman TH. Diagnósticos de enfermagem da NANDA: definições e classificação 2012-2014. Porto Alegre: Artmed; 2013.

2. Ministério da Saúde (BR). Portaria n. 529, de 1 de abril de 2013. Institui o Programa Nacional de Segurança do Paciente (PNSP). Diário Oficial da União, [Internet] 02 abr 2013 [acesso em 14 mai 2014]. Disponível: http://bvsms.saude.gov.br/bvs/ saudelegis/gm/2013/prt0529_01_04_2013.html.

3. da Costa RG, Vitor AF, Botarelli FR, Moura LA, Vitorino ICC, Soares CS. Diagnósticos de enfermería del Dominio de Seguridad/ protección en pacientes en postoperatorio. Rev Cubana enfermer. [Internet] 2014; 30(4) [acesso em 14 jan 2016]. Disponível: http://www.revenfermeria.sld.cu/index.php/enf/article/view/504/104.

4. Mangueira SO, Fernandes AFC, Pinheiro AKB, Lopes MVO. Indicadores clínicos do diagnóstico de enfermagem processos familiares disfuncionais em alcoolistas: revisão integrativa. Rev. eletrônica enferm. [Internet] 2013; 15(3) [acesso em 14 mai 2014]. Disponível: http://dx.doi.org/10.5216/ree.v15i3.17766.

5. Maluf-Filho FA. Contribuição da medicina baseada em evidências para a introdução de novo conhecimento na prática clínica. Arq. gastroenterol. [Internet] 2009; 46(2) [acesso em 13 jan 2016]. Disponível: http://dx.doi.org/10.1590/S000428032009000200002.

6. Mendes KDS, Silveira RCCP, Galvao CM. Revisão integrativa: método de pesquisa para a incorporação de evidências na saúde e na enfermagem. Texto Contexto Enferm. [Internet] 2008; 17(4) [acesso em 12 jan 2016]. Disponível: http://dx.doi. org/10.1590/S0104-07072008000400018.

7. Olsen J. Meta-analysis or Collaborative Studies. J. Occup. Environ. Med. 1995;37(8):897-902.

8. de Oliveira AC, Kovner CT, da Silva RS. Infecção hospitalar em unidade de tratamento intensivo de um hospital universitário brasileiro. Rev. Latino-Am. Enfermagem. [Internet] 2010; 18(2) [acesso em 12 jan 2016]. Disponível: http://dx.doi.org/10.1590/ S0104-11692010000200014.

9. Roque KE, Melo ECP. Tempo de internação e a ocorrência de eventos adversos a medicamentos: uma questão de enfermagem. Esc. Anna Nery. [Internet] 2011; 15(3) [acesso em 13 jan 2016]. Disponível: http://dx.doi.org/10.1590/S141481452011000300022.

10. Gomes FSL, Bastos MAR, Matozinhos FP, Temponi HR, Velásquez-Meléndez G. Fatores associados à úlcera por pressão em 
pacientes internados nos Centros de Terapia Intensiva de Adultos. Rev. esc. enferm. USP. [Internet] 2010; 44(4) [acesso em 15 jan 2016]. Disponível: http://dx.doi.org/10.1590/S0080-62342010000400031.

11. Sales MVC, Silva TJA, Gil Júnior LA, Jacob Filho W. Efeitos adversos da internação hospitalar para o idoso. Geriatr. Gerontol. 2010; 4(4): 238-46.

12. Vargas e Silva MR, Dick NRM, Martini AC. Incidência de úlcera por pressão como indicador de qualidade na assistência de enfermagem. Rev enferm UFSM. [Internet] 2012; 2(2) [acesso em 15 jan 2016]. Disponível: http://dx.doi. org/10.5902/217976925238.

13. Cremasco MF, Wenzel F, Zanei SV, Whitaker IY. Pressure ulcers in the intensive care unit: the relationship between nursing workload, illness severity and pressure ulcer risk. J Clin Nurs. [Internet] 2012; 22(15-16) [acesso em 13 jan 2016 ]. Disponível: http://dx.doi.org/10.1111/j.1365-2702.2012.04216.x.

14. Gonçalves LA, Andolhe R, de Oliveira EM, Barbosa RL, Mancussi e Faro AC, Gallotti RMD, et al. Alocação da equipe de enfermagem e ocorrência de eventos adversos/incidentes em unidade de terapia intensiva. Rev. esc. enferm. USP. [Internet] 2012; 46(n.esp) [acesso em 16 jan 2016]. Disponível: http://dx.doi.org/10.1590/S0080-62342012000700011.

15. Lopes AER, Pompeo DA, Canini SRMS, Rossi LA. Diagnósticos de enfermagem de pacientes em período pré-operatório de cirurgia esofágica. Rev. Latino-Am. Enfermagem. [Internet] 2009; 17(1) [acesso em 16 jan 2016]. Disponível: http://dx.doi. org/10.1590/S0104-11692009000100011.

16. Organização Mundial da Saúde (OMS). Caídas. [Internet] 2012 [acesso em 27 out 2015]. Disponível: www.who.int/ mediacentre/factsheets/fs344/es/.

17. Abreu C, Mendes A, Monteiro J, Santos FR. Quedas em meio hospitalar: um estudo longitudinal. Rev. Latino-Am. Enfermagem. [Internet] 2012; 20(3) [acesso em 12 jan 2016]. Disponível: http://dx.doi.org/10.1590/S0104-11692012000300023.

18. Correa AD, Marques IAB, Martinez MC, Laurino PS, Leão ER, Chimentão DMN. Implantação de um protocolo para gerenciamento de quedas em hospital: resultados de quatro anos de seguimento. Rev. esc. enferm. USP. [Internet] 2012; 46(1) [acesso em 16 jan 2016]. Disponível: http://dx.doi.org/10.1590/S0080-62342012000100009.

19. da Silva LD, Camerini FG. Análise da administração de medicamentos intravenosos em hospital da rede sentinela. Texto Contexto Enferm. [Internet] 2012; 21(3) [acesso em 12 jan 2016]. Disponível: http://dx.doi.org/10.1590/S010407072012000300019. 